\title{
ROBUST SEMIGLOBAL STABILIZATION OF THE SECOND ORDER SYSTEM BY RELAY FEEDBACK WITH AN UNCERTAIN VARIABLE TIME DELAY*
}

\author{
EUGENII SHUSTIN ${ }^{\dagger}$, LEONID FRIDMAN $^{\ddagger}$, EMILIA FRIDMAN ${ }^{\S}$, AND \\ FERNANDO CASTAÑOS
}

\begin{abstract}
We present sufficient conditions for robust relay-delayed semiglobal stabilization of second order systems, which relate the upper bound to an uncertain time delay and the parameters of the plant. We also suggest an algorithm of delayed relay control gain adaptation for semiglobal stabilization, which is based on delayed information about the sign of the controlled variable only. The proposed algorithm suppresses bounded uncertainties in the time delay; that is, being designed for the upper bound of uncertainty in the time delay, the control law ensures semiglobal stabilization independently of any variable time delay obeying the given upper bound.
\end{abstract}

Key words. variable structure systems, time delay, robust control

AMS subject classifications. 34K20, 34K35, 93B12, 93B51, 93D09

DOI. $10.1137 / 060673333$

\section{Introduction.}

1.1. Statement of the problem. We study the control problem for the second order system

$$
\alpha \ddot{x}(t)=-\beta \dot{x}(t)+F(x(t), t)+u,
$$

with positive constants $\alpha$ and $\beta$ and some function $F(x, t)$, satisfying

$$
F \in C^{1}\left(R^{2}\right), \quad \sup \left|\frac{\partial F}{\partial x}\right|<\infty
$$

The uncontrolled system

$$
\alpha \ddot{x}=-\beta \dot{x}+F(x, t)
$$

may be unstable, as, for example, in the case $F(x, t)=k x, k>0$, and we propose to stabilize it by a negative feedback of relay type:

$$
u=-K(t) \cdot \operatorname{sign} x(t-\tau),
$$

*Received by the editors October 25, 2006; accepted for publication (in revised form) June 26, 2007; published electronically January 11, 2008.

http://www.siam.org/journals/sicon/47-1/67333.html

†School of Mathematical Sciences, Tel Aviv University, Ramat Aviv, 69978 Tel Aviv, Israel (shustin@post.tau.ac.il).

${ }^{\ddagger}$ Department of Control, Division of Electrical Engineering, Engineering Faculty, National Autonomous University of Mexico, UNAM, 04510, Mexico, D.F., Mexico (Ifridman@servidor.unam.mx, http://verona.fi-p.unam.mx/ 1 fridman/).

$\S$ Department of Electrical Engineering and Systems, Tel Aviv University, Ramat Aviv, $69978 \mathrm{Tel}$ Aviv, Israel (emilia@eng.tau.ac.il).

『Laboratoire des Signaux et Systèmes, Supélec, 3 rue Joliot Curie, 91192 Gif-sur-Yvette, France (castanos@lss.supelec.fr). 
with a controllable bounded magnitude $K(t)>0$ and a positive variable uncertain delay $\tau$, assumed to be a measurable function of $t$ obeying the condition

$$
0<\tau_{0}(t) \leq \tau(t) \leq h=\text { const }, \quad t \geq 0,
$$

where $\tau_{0}(t)$ is a positive nonincreasing function.

Our aim is to design a piecewise constant controller $K(t)$, which provides a robust semiglobal stabilization of the oscillation magnitude of the solutions to system (1), (3).

1.2. Motivation. For the motivation, we point out that time delay in control systems is usually present and must be taken into account. In practice, many systems with time delay naturally admit relay controllers, in particular,

- systems which can work in switching modes, for example, power converters (see, for example, [19]);

- systems with measuring devices that work in the switching mode and have time delay, for example, controllers of exhausted gas in the fuel injector automotive control systems [15], which act with delay and, moreover, generate relay signals only;

- sliding mode systems with delayed actuators, for example, the stabilizers of the fingers for an underwater manipulator [2];

- mathematical biology systems as, for example, those considered in [12, 13].

It has been shown in $[5,6]$ that, in the simplest one-dimensional relay control systems with a constant delay, only oscillatory solutions can occur. Moreover, any such solution becomes periodic after a finite time interval, but only slowly oscillating solutions are stable. The latter property is used to design an algorithm controlling the motion amplitudes.

P.I. (proportional-integral) control algorithms for the amplitude control in onedimensional relay systems with delay in the input have been suggested in [1]. A Padé approximation of delay that reduces the relay delay output tracking problem to the sliding mode control for a nonminimum phase system was suggested in [16]. Delayed relay control algorithms, suggested in $[7,8]$, allow one to reach local and nonlocal stabilization of oscillations amplitudes for MIMO systems, respectively, with the use of the delayed value of the magnitude of a current trajectory.

In [11], periodic properties of second order systems via relay-delayed controllers based on the suboptimal control algorithm were investigated, whereas the article [3] studies oscillations in first order systems, containing external forcing in the relaydelayed control element.

1.3. The main result. Restrictions to the nonlinear element. Throughout the paper we impose the following bound of the nonlinear term $F(x, t)$ of (1):

$$
0 \leq \frac{F(x, t)-F(0, t)}{x} \leq k_{0}, \quad x \neq 0, \quad t \geq 0,
$$

with some positive constant $k_{0}$. Furthermore, we separate between the two situations:

$$
F(0, t) \equiv 0,
$$

and

$$
|F(0, t)| \leq \delta, \quad t \geq 0, \quad \delta=\text { const } \in(0,1)
$$


in which the suggested controller and the respective solutions to (1) and (3) behave differently.

The initial value problem and the definition of the discontinuous element. For system (1), (3), we state the initial value problem

$$
x(t)=\varphi(t), t \in[-h, 0], \varphi \in C_{0}[-h, 0], \quad \dot{x}(0)=\dot{\varphi}(0),
$$

by defining the initial data range to be the space $C_{0}[-h, 0]$ of continuous functions $\varphi:[-h, 0] \rightarrow \mathbb{R}$, differentiable at the origin. We equip $C_{0}[-h, 0]$ with the norm

$$
\|\varphi\|=\max _{[-h, 0]}|\varphi(t)|+|\dot{\varphi}(0)| .
$$

The fact that the functions $\varphi \in C_{0}[-h, 0]$ may vanish along intervals raises an important issue of an appropriate choice of the values of the sign function at vanishing arguments. From the control theory point of view, sign should be a binary sensor or actuator; i.e., it takes the only values \pm 1 . So we will define

$$
\operatorname{sign} x(t)= \begin{cases}1 & \text { if } x(t)>0, \\ -1 & \text { if } x(t)<0, \\ \zeta(t) & \text { if } x(t)=0,\end{cases}
$$

where $\zeta(t)$ is any measurable function with $|\zeta(t)|=1$, and consider the solutions to system (1), (3) in the sense of Carathéodory (see, for example, [9]). ${ }^{1}$

Then we have the following.

Lemma 1.1. The equation

$$
\alpha \ddot{x}(t)=-\beta \dot{x}(t)+F(x(t), t)-\operatorname{sign} x(t-\tau),
$$

satisfying (2), (4), and (7), with initial condition (8) supplied with (10), has a unique continuous solution $x_{\varphi}(t), t \in[-h, \infty)$. Moreover, $x_{\varphi}$ is differentiable in the interval $(0, \infty)$, and its derivative is absolutely continuous and differentiable almost everywhere.

We omit the proof, which basically coincides with the proof of Lemma 1.1 in [17], and notice only that the lower bound to $\tau$ in (4) is needed for an accurate justification of the existence and uniqueness of the solution $x_{\varphi}$.

Remark 1. (i) The solutions $x(t)$ to system (1), (3) considered in what follows will satisfy the condition $|F(x(t), t)|<K(t)$ (cf. the combination of bounds (5), (7), and (20) and of Lemmas A.1, A.2, and A.3 below), and thus, in the same way as in Lemma 1.1, the zero locus of such a solution $x(t)$ in the interval $t \geq 0$ will have zero measure whatever the zero locus of the initial function $\varphi(t) \in C_{0}[-h, 0]$ is, and hence the results do not depend on the choice of the function $\zeta(t)$ in (10) for $t \geq 0$. In particular, shifting the initial interval to $[0, h]$, one obtains the zero locus of zero measure for the (new) initial function, getting rid of any dependence of the function $\zeta(t)$. In addition, $\dot{x}(t)$ turns out to be differentiable almost everywhere.

(ii) The Filippov differential inclusion theory [4] commonly used for nondelayed differential equations with discontinuity and intended to turn solutions into sliding

\footnotetext{
${ }^{1}$ The sliding modes cannot occur in the considered class of the systems. That is why it is not necessary to use more complicated definitions of the solutions for relay systems with delay (see, for example, $[10,14])$.
} 
modes, i.e., motions along the discontinuity locus (see detailed accounts in [10] and [14]), is not quite relevant in our situation. Indeed, the motion along the discontinuity locus should correspond to the zero solution $x(t) \equiv 0$, which cannot be stable. The reason is that, in the norm (9), the zero function $\varphi(t) \equiv 0$ can be approximated by functions with at most one zero, which in turn generate either unbounded solutions or solutions with the sup-norm separated from zero (see, for example, [17]). On the other hand, an attempt to keep the zero level of an eventually vanishing solution leads to the relation

$$
\left.(\operatorname{sign} x(t-\tau))\right|_{x(t-\tau)=0}=F(x(t), t),
$$

which is not natural for a controller based on only delayed information.

The statement. We provide here a general statement, solving the stated problem, and leave the precise formulation for section 3.

Main result. Given system (1), (3) with $F(x, t)$ satisfying (5), under certain restrictions to $\alpha, \beta, h, \delta$, and $k_{0}$, there exist positive constants $c, T_{0}, m$, and $\rho<1$ such that

(i) in the case (6), for

$$
K(t)=\rho^{n}, \quad n T_{0} \leq t<(n+1) T_{0}, \quad n=0,1,2, \ldots,
$$

all of the solutions with $\max \{|x(0)|,|\dot{x}(0)|\}<c$ exponentially decay to zero;

(ii) in the case (7), for

$$
K(t)= \begin{cases}\rho^{n}, & n T_{0} \leq t<(n+1) T_{0}, \quad n=0,1,2, \ldots, m-1, \\ \rho^{m}, & t \geq m T_{0},\end{cases}
$$

all of the solutions with $\max \{|x(0)|,|\dot{x}(0)|\}<c$ come to a neighborhood of zero, whose size is proportional to $\delta$.

In section 3.1, we provide explicit formulas for all of the parameters $\alpha, \beta, h, \delta$, and $k_{0}$, and in section 5 , we make a numerical simulation.

The meaning of main result is that, whenever the parameters of the system (1) and the controller delay $\tau$ satisfy some explicitly written restriction, a control presented by a step function $K(t)$ with a priori fixed switch moments and amplitudes brings solutions to a prescribed neighborhood of zero. In other words, we propose an algorithm for a robust semiglobal stabilization of the oscillation magnitude, based on a retarded relay switching of the control gain, which requires only the knowledge of the sign for the controlled variable in the past and allows us to reject uncertainty in the time delay.

1.4. The ideas behind the main result. The idea of a piecewise constant control function $u(t)$ can be traced back to [6], where such a controller, acting with a constant delay, has been used for an exponential stabilization of oscillations in the first order system

$$
\dot{x}(t)=F(x(t), t)-\operatorname{sign} x(t-h),
$$

with $F$ satisfying (5) and (6). The key observation was that, if $k_{0} h<\log 2$, the solutions starting in a small neighborhood of zero cannot reach some critical value $|x|=M_{0}$ during the time interval $h$ and then must return to the zero level, that is, remain bounded and oscillating. Furthermore, for such solutions, sup $|x(t)|<M_{1}<$ 
$M_{0}$, and hence, switching the magnitude of sign from 1 to $\rho=M_{1} / M_{0}<1$ at the moment $t^{*}$, with $x\left(t^{*}\right)=0$, and making change $x=\rho x^{(1)}$, we come to an equation

$$
\dot{x}^{(1)}(t)=F^{(1)}\left(x^{(1)}(t), t\right)-\operatorname{sign} x^{(1)}(t-h),
$$

with $F^{(1)}$ again satisfying (5) and (6), which in turn means $|x(t)|<\rho M_{0}$ as $t \geq$ $t^{*}$. Performing inductively the same procedure, one obtains exponentially decreasing solutions. However, that controller was depending on the term $F(x, t)$ and on the current solution, which made it hard to realize in practice. This difficulty has been resolved in [18], where a similar piecewise constant controller acting with a variable uncertain bounded delay and having a priori fixed switches provided an exponential decay of solutions with sufficiently small initial values.

In a similar way we obtain the main result for the second order system (1), (3). The background property, established in [17], states that, under certain restrictions on the positive parameters $\alpha, \beta, k, K, h, c$, the solutions to the equation

$$
\alpha \ddot{x}=-\beta \dot{x}+k x-K \cdot \operatorname{sign} x(t-h),
$$

which obey the initial conditions $x(0)=0,|\dot{x}(0)|<c$, remain bounded by a constant $M$, proportional to $K$, and, moreover, the derivatives $\dot{x}\left(t^{*}\right)$ for all $t^{*}>0, x\left(t^{*}\right)=0$, belong to a smaller range $\left(-c_{1}, c_{1}\right)$, where $c_{1}<c$. Here we extend this fact to the case of arbitrary functions $F(x, t)$ with bounded values and derivative and a variable uncertain delay $\tau(t)$. So, again after a suitable period of time, we switch the controller magnitude from $K$ to $\rho K$, with some $\rho \in\left(c_{1} / c_{0}, 1\right)$, and make change $x=\rho x^{(1)}$, coming to an equation for $x^{(1)}$, analogous to (1), (3) and satisfying the hypotheses, which provide $\left|x^{(1)}\right|<M$ and $\left|\dot{x}^{(1)}\right|<c$, and, in particular, $|x(t)|<\rho M$ for large $t>0$.

To find suitable bounds to the given data, we model the "worst" behavior of a solution to (1), (3), which means that the absolute value $|x|$ maximally grows against the negative feedback $u$ intended to bring the solution to the zero level. That is, if a solution starts at zero with some, say, positive derivative, we assume that $F(x, t)=\delta+k x$ and $\tau=h$, so that the feedback $u$ remains positive on the largest possible interval of length $h$. Then we assume that the value of the control undergoes a switch only after a period of time $h$ has elapsed, and we know that reversing the sign of the control will eventually force the solution to reach a maximum. When the maximum value is attained, we take $F(x, t)=-\delta$ and wait until the solution reaches the next zero. We call the pieces of that worst solution majorating functions. They are treated in the next section in order to precisely state the sufficient conditions for the existence of the controller proposed in the main result, and these conditions finally reduce to the claim that the absolute value of the derivative of the worst solution at its zero is strictly greater than that value at the next zero.

\section{Majorating functions.}

2.1. Definition of the majorating functions. First, we point out that restriction (5) on the nonlinearity $F(x, t)$ comes from the comparison of system (1), (3) with the equation $\alpha \ddot{x}=-\beta \dot{x}+k_{0} x-\operatorname{sign} x(t-\tau)$, and this makes it natural to introduce the roots $\lambda_{1}>0>\lambda_{2}$ of the characteristic equation $\alpha \lambda^{2}+\beta \lambda-k_{0}=0$, which will play an important role in the further consideration.

In order to deal with uncertainty, we introduce a majorating function for the actual response $x(t)$. This function is intended to model "the worst type of behavior" of the stable solutions to (1), (3). As previously stated, such solutions are periodic 


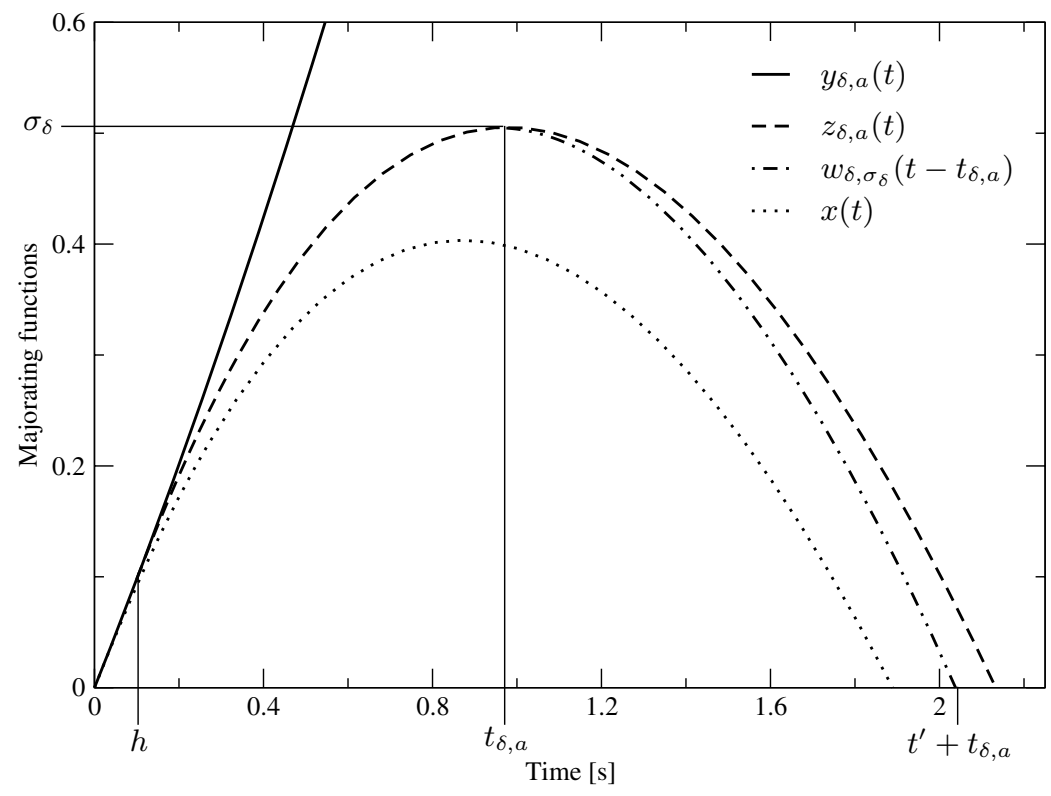

FIG. 1. Actual response $x(t)$ and the majorating functions.

and slow. In Figure 1 we show (as a dotted line) the upper lobe of one of the periods. Assuming that the distance between the neighboring zeros of $x(t)$ is greater than $h$, we can divide the interval between such zeros into three parts:

1. an interval between the current zero and the (first) control switch,

2. an interval between the control switch and the global extremum,

3. the remaining part from the extremum to the next zero.

For the first interval consider the equation

$$
\alpha \ddot{y}(t)=-\beta \dot{y}(t)+k_{0} y(t)+1+\delta .
$$

We assume that the control switch is delayed by $h$ from the current zero, and, since we are considering the upper lobe, the initial derivative is positive. Hence we impose

$$
0 \leq t \leq h, \quad y(0)=0, \dot{y}(0)=a,
$$

where $a$ is a nonnegative parameter.

The family of functions $y_{\delta, a}(t)$ (see Figure 1)

$$
y_{\delta, a}(t)=\frac{a k_{0}-\lambda_{2}(1+\delta)}{k_{0}\left(\lambda_{1}-\lambda_{2}\right)} e^{\lambda_{1} t}+\frac{\lambda_{1}(1+\delta)-a k_{0}}{k_{0}\left(\lambda_{1}-\lambda_{2}\right)} e^{\lambda_{2} t}-\frac{1+\delta}{k_{0}}
$$

are the solutions of the previous equation.

For the second interval we introduce the solution

$$
\begin{gathered}
z_{\delta, a}(t)=-\frac{2 e^{-\lambda_{1} h}-1-\delta-\alpha a \lambda_{1}}{\alpha \lambda_{1}\left(\lambda_{1}-\lambda_{2}\right)} e^{\lambda_{1} t}+\frac{2 e^{-\lambda_{2} h}-1-\delta-\alpha a \lambda_{2}}{\alpha \lambda_{2}\left(\lambda_{1}-\lambda_{2}\right)} e^{\lambda_{2} t} \\
+\frac{1-\delta}{k_{0}}
\end{gathered}
$$

Copyright $\odot$ by SIAM. Unauthorized reproduction of this article is prohibited. 
of the equation

$$
\alpha \ddot{z}(t)=-\beta \dot{z}(t)+k_{0} z(t)-1+\delta, \quad z(h)=y_{\delta, a}(h), \dot{z}(h)=\dot{y}_{\delta, a}(h) .
$$

Suppose that $2 e^{-\lambda_{1} h}-1>0$,

$$
\delta<2 e^{-\lambda_{1} h}-1
$$

and

$$
a<\frac{2 e^{-\lambda_{1} h}-1-\delta}{\alpha \lambda_{1}}
$$

This means, in particular, that the coefficients of $e^{\lambda_{1} t}$ and $e^{\lambda_{2} t}$ in (13) are negative. Hence $z_{\delta, a}(t)$ is a concave function, which in view of $\dot{z}_{\delta, a}(h)=\dot{y}_{\delta, a}(h)>0$ has a unique maximum in $(h, \infty)$. The maximum occurs at the time moment

$$
t_{\delta, a}=\frac{1}{\lambda_{1}-\lambda_{2}} \log \frac{2 e^{-\lambda_{2} h}-1-\delta-\alpha a \lambda_{2}}{2 e^{-\lambda_{1} h}-1-\delta-\alpha a \lambda_{1}}
$$

so for $z_{\delta, a}(t)$ we add the restriction

$$
h \leq t \leq t_{\delta, a} .
$$

To keep the notation simple, we will set

$$
\sigma_{\delta} \triangleq z_{\delta, a}\left(t_{\delta, a}\right) .
$$

For the last interval we introduce the equation

$$
\alpha \ddot{w}(t)=-\beta \dot{w}(t)-1-\delta .
$$

In what follows, we will be more concerned about the value of the global extremum $\sigma_{\delta}$, rather than the time of its occurrence $t_{\delta, a}$, so we add

$$
w(0)=\sigma_{\delta}, \quad \dot{w}(0)=0,
$$

which defines a majorating function which is shifted in time (see Figure 1). The solution is given by

$$
w_{\delta, \sigma_{\delta}}(t)=\frac{\alpha(1+\delta)}{\beta^{2}}\left(1-e^{-t \beta / \alpha}\right)-\frac{1+\delta}{\beta} t+\sigma_{\delta},
$$

which has a unique positive root $t^{\prime}$ (see Figure 2).

Now we can build the majorating function. For any perturbation with a bound $\delta$ satisfying (14) and an initial derivative $a$ satisfying (15), the function

$$
\phi_{\delta, a}(t) \triangleq \begin{cases}y_{\delta, a}(t), & 0 \leq t \leq h, \\ z_{\delta, a}(t), & h \leq t \leq t_{\delta, a}, \\ w_{\delta, \sigma_{\delta}}\left(t-t_{\delta, a}\right), & t_{\delta, a} \leq t \leq t^{\prime}+t_{\delta, a},\end{cases}
$$

bounds from above the solutions to (1), (3) and their derivatives. We shall call $\phi_{\delta, a}(t)$ the worst solution to (1), (3). 

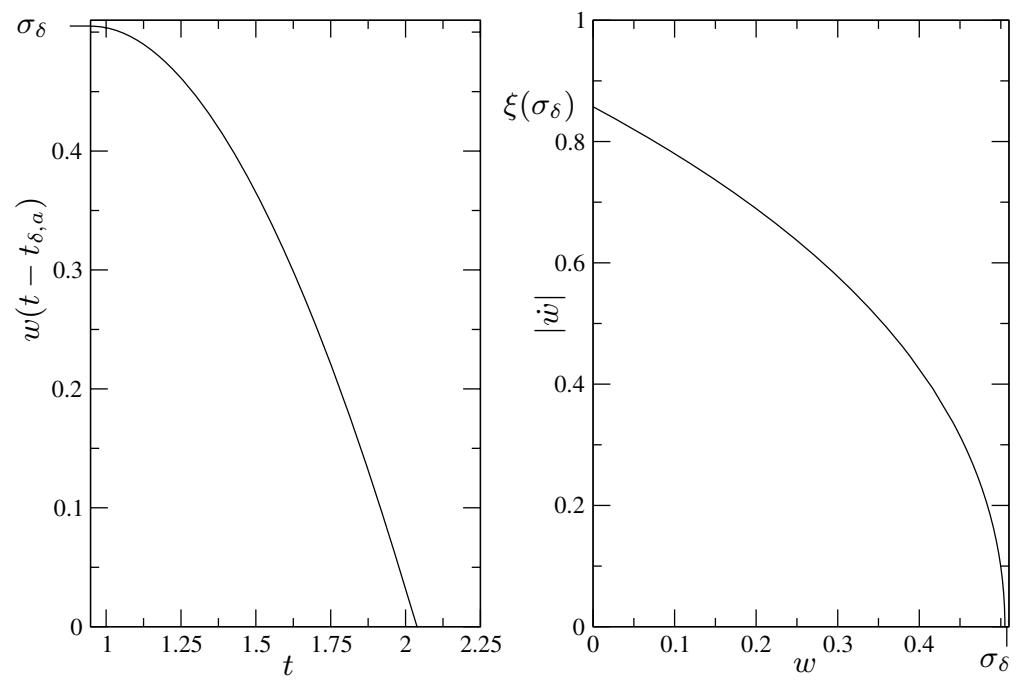

FIG. 2. Behavior of the last majorating function $w\left(t-t_{\delta, a}\right)$.

2.2. Properties of the majorating functions. The next property will play a key role in the argumentation that follows.

Definition 2.1. A function $\phi_{a}(t) \in C_{1}$ is said to be differentially contractive $(D C)$ if, whenever it starts at a zero with a derivative belonging to some interval, its derivative at the next zero belongs to a smaller interval.

Notice that $\phi_{\delta, a}$ is continuous (see Figure 1). Its initial derivative $a$ is taken from the interval (15), and we want its terminal derivative

$$
\xi_{\delta}\left(\sigma_{\delta}\right) \triangleq\left|\dot{w}_{\delta, \sigma_{\delta}}\left(t^{\prime}\right)\right|
$$

to belong to a smaller interval.

To fulfill the DC property, we first use (18) to estimate $t^{\prime}$

$$
\frac{\alpha(1+\delta)}{\beta^{2}}\left(1-e^{-t^{\prime} \beta / \alpha}\right)=\frac{1+\delta}{\beta} t^{\prime}-\sigma_{\delta}
$$

Due to $\dot{z}_{\delta, a}\left(t_{\delta, a}\right)=0$ and $\ddot{z}_{\delta, a}\left(t_{\delta, a}\right)<0$, we have

$$
\sigma_{\delta}=\frac{1-\delta+\beta \dot{z}_{\delta, a}\left(t_{\delta, a}\right)+\alpha \ddot{z}_{\delta, a}\left(t_{\delta, a}\right)}{k_{0}}<\frac{1-\delta}{k_{0}} .
$$

Hence $t^{\prime} \leq \theta$, where $\theta$ is the positive root of the equation

$$
\frac{\alpha(1+\delta)}{\beta^{2}}\left(1-e^{-\theta \beta / \alpha}\right)=\frac{1+\delta}{\beta} \theta-\frac{1-\delta}{k_{0}} .
$$

Notice that, given $\alpha, \beta, \delta, k_{0},(21)$ always has a unique positive root $\theta$, since the lefthand side is a positive concave function of $\theta$ and the right-hand side is an increasing linear function of $\theta$, negative at the origin. Next, we have that

$$
\xi_{\delta}\left(\sigma_{\delta}\right)=\frac{1+\delta}{\beta}\left(1-e^{-t^{\prime} \beta / \alpha}\right) \leq \frac{1+\delta}{\beta}\left(1-e^{-\theta \beta / \alpha}\right) .
$$

Copyright (c) by SIAM. Unauthorized reproduction of this article is prohibited. 
In view of the last inequality and (15) it is easy to see that $\phi_{\delta, a}$ fulfills the DC property if

$$
\frac{1+\delta}{\beta}\left(1-e^{-\theta \beta / \alpha}\right)<\frac{2 e^{-\lambda_{1} h}-1-\delta}{\alpha \lambda_{1}} .
$$

To understand inequality (23), consider the equality

$$
\frac{1+\delta}{\beta}\left(1-e^{-\theta(k) \beta / \alpha}\right)=\frac{2 e^{-\lambda_{1}(k) h}-1-\delta}{\alpha \lambda_{1}(k)}
$$

as an equation to the unknown $k$ with fixed $\alpha, \beta, \delta$. Here the left-hand side is a bounded positive function of $k$, whereas the right-hand side drops from infinity to negative values as $k$ grows from zero to infinity. Hence (24) has positive roots, ${ }^{2}$ and the minimal one among them we denote by $k_{\min }$. So, finally, we reduce (23) to

$$
k_{0}<k_{\min }
$$

which guarantees the DC property.

Remark 2. According to (20) the extremum $\sigma_{\delta}$ is bounded from above. We shall call that bound $\sigma_{\max }$, i.e.,

$$
\sigma_{\delta}<\sigma_{\max } \triangleq \frac{1-\delta}{k_{0}}
$$

Suppose that the extremum attains the maximum value in the current period; in view of (20), the extremum at the following period satisfies

$$
\sigma_{\delta}^{(1)} \triangleq z_{\delta, \xi_{\delta}\left(\sigma_{\max }\right)}\left(t_{\xi_{\delta}\left(\sigma_{\max }\right)}\right)<\sigma_{\max }
$$

\section{Main results in detail.} either

Definition 3.1. Denote by $\Phi_{\delta, a}$ the set of functions $\varphi \in C_{0}[-h, 0]$ such that

$$
\varphi^{-1}(0) \neq \emptyset, \quad|\varphi(0)| \leq y_{\delta, a}\left(-t^{*}\right), \quad|\dot{\varphi}(0)| \leq \dot{y}_{\delta, a}\left(-t^{*}\right),
$$

where $t^{*}=\max \varphi^{-1}(0)>-h$, or

$$
\left.\varphi\right|_{(-h, 0]} \neq 0, \quad|\varphi(0)| \leq z_{\delta, a}\left(t^{*}\right), \quad|\dot{\varphi}(0)| \leq \dot{z}_{\delta, a}\left(t^{*}\right)
$$

for some $t^{*} \in\left[h, t_{\delta, a}\right]$.

3.1. Perturbations that vanish at the origin. Assume that $\delta=0$. In order to simplify the notation, in this case we always skip the subindex $\delta$ (i.e., 0 ) in the notation for $t, \xi, \sigma, \Phi, x, y, z$.

Introduce the following parameter. Given

$$
0<a<b<\frac{2 e^{-\lambda_{1} h}-1}{\alpha \lambda_{1}}
$$

set

$$
\rho(a, b) \triangleq \frac{\alpha a\left(\lambda_{1} e^{\lambda_{1} h}-\lambda_{2} e^{\lambda_{2} h}\right)+\left(e^{\lambda_{1} h}-e^{\lambda_{2} h}\right)}{\alpha b\left(\lambda_{1} e^{\lambda_{1} h}-\lambda_{2} e^{\lambda_{2} h}\right)+\left(e^{\lambda_{1} h}-e^{\lambda_{2} h}\right)}
$$

\footnotetext{
${ }^{2}$ It is an easy exercise to show that a positive root is unique, but we shall not need this fact.
}

Copyright $@$ by SIAM. Unauthorized reproduction of this article is prohibited. 
Clearly, $\rho(a, b)<1$. Then introduce

$$
\rho \triangleq \rho\left(\xi\left(\sigma^{(1)}\right), \xi\left(\sigma_{\max }\right)\right) .
$$

Notice that $\rho$ is defined properly, since $\xi$ is a strictly increasing function.

TheOREM 1. Assume that $F(x, t)$ and $\tau(t)$ satisfy $(2),(4),(5),(6)$, and $(25)$ with $\delta=0$. Let a constant $c$ satisfy

$$
0<c<\frac{2 e^{-\lambda_{1} h}-1}{\alpha \lambda_{1}} .
$$

Put

$$
K(t)=\left\{\begin{array}{rll}
1 & \text { if } \quad & 0 \leq t<t_{c}, \\
\rho^{n} \quad \text { if } \quad & t_{c}+n t_{\xi\left(\sigma_{\max }\right)} \leq t<t_{c}+(n+1) t_{\xi\left(\sigma_{\max }\right)}, \\
& & n=0,1,2, \ldots,
\end{array}\right.
$$

where $\rho$ is defined by $(27)$, and $t_{c}$ and $t_{\xi\left(\sigma_{\max }\right)}$ are the roots of $z_{c}(t)$ and $z_{\xi\left(\sigma_{\max }\right)}(t)$, respectively (see Figure 1).

Then any solution $x_{\varphi}(t)$ to $(1),(3),(8)$ with $\varphi \in \Phi_{c}$ obeys the restriction

$$
\left|x_{\varphi}(t)\right| \leq \frac{1}{k_{0}} \exp \left(-\left(\log \frac{1}{\rho}\right) \frac{t-t_{c}-t_{\xi_{\delta}\left(\sigma_{\max }\right)}}{t_{\xi_{\delta}\left(\sigma_{\max }\right)}}\right), \quad t \geq t_{c}+t_{\xi_{\delta}\left(\sigma_{\max }\right)} .
$$

3.2. Perturbations that do not vanish at the origin. In realistic models, $F(0, t)$ does not vanish identically, so we'll consider the case $\delta \neq 0$, but we'll maintain restriction (14). In this case, one can drive the system in a finite time to a neighborhood of zero, proportional to $\delta$. More precisely, we design a set of controllers, which depend on one continuous and one discrete parameter. The parameters can be chosen in their range according to the initial magnitude, the required rate of convergence, and the size of the target neighborhood of zero. We remark only that one cannot optimize the two latter values simultaneously.

Given $\delta$ satisfying (14), the range of a positive parameter $\varepsilon$ is defined by the inequality

$$
\frac{1+\delta}{\beta}\left(1-e^{-\theta \beta / \alpha}\right)+\frac{\delta}{\alpha \lambda_{1}}<\frac{2 e^{-\lambda_{1} h}-1-\varepsilon}{\alpha \lambda_{1}} .
$$

Observe that (30) defines a nonempty interval, since it turns into (23) for $\varepsilon=0$. Next we choose any natural $m \geq 1$ and put $q=q(\varepsilon, m)$ to be the positive root of the equation

$$
\begin{aligned}
\frac{1}{q}\left(\frac{1-e^{-\theta \beta / \alpha}}{\beta}+\frac{(1-q)\left(e^{\lambda_{1} h}-e^{\lambda_{2} h}\right)}{\alpha\left(\lambda_{1} e^{\lambda_{1} h}-\lambda_{2} e^{\lambda_{2} h}\right)}\right)+\frac{\delta}{q^{m}}\left(\frac{1-e^{-\theta \beta / \alpha}}{\beta}\right. & \left.+\frac{1}{\alpha \lambda_{1}}\right) \\
& =\frac{2 e^{-\lambda_{1} h}-1-\varepsilon}{\alpha \lambda_{1}}
\end{aligned}
$$

Such a root does exist; furthermore, it is unique and belongs to the interval $(0,1)$. Indeed, the left-hand side of (31) monotonically decreases from infinity to the left-hand 
side of (30), whereas the right-hand sides of (30) and (31) coincide. Furthermore,

$$
\begin{array}{r}
\frac{1}{q}\left(\frac{1-e^{-\theta \beta / \alpha}}{\beta}+\frac{(1-q)\left(e^{\lambda_{1} h}-e^{\lambda_{2} h}\right)}{\alpha\left(\lambda_{1} e^{\lambda_{1} h}-\lambda_{2} e^{\lambda_{2} h}\right)}\right)+\frac{\delta}{q^{m^{\prime}}}\left(\frac{1-e^{-\theta \beta / \alpha}}{\beta}\right. \\
\left.+\frac{1}{\alpha \lambda_{1}}\right) \\
\leq \frac{2 e^{-\lambda_{1} h}-1-\varepsilon}{\alpha \lambda_{1}}
\end{array}
$$

for all $m^{\prime} \leq m$. At last, put

$$
T(\varepsilon)=\frac{1}{\lambda_{1}} \log \frac{(1-\delta)\left(\lambda_{1}-\lambda_{2}\right)}{-\lambda_{2} \varepsilon} .
$$

Theorem 2. Under the hypotheses (2), (4), (5), (7), and (25) with $\delta>0$ satisfying (14), let $\varepsilon$ obey (30). Put

$$
K(t)= \begin{cases}q^{s} \quad \text { if } \quad s T(\varepsilon) \leq t<(s+1) T(\varepsilon), s=0,1, \ldots, m-1, \\ q^{m} \quad \text { if } \quad t \geq m T(\varepsilon) .\end{cases}
$$

Then any solution $x_{\varphi}(t)$ to $(1),(3),(8)$, with $\varphi \in \Phi_{\delta, c}$, where

$$
c=\frac{2 e^{-\lambda_{1} h}-1-\delta-\varepsilon}{\alpha \lambda_{1}},
$$

obeys the restriction

$$
\left|x_{\varphi}(t)\right| \leq \frac{q^{m}-\delta}{k_{0}} \quad \text { as } \quad t \geq m T(\varepsilon) .
$$

\section{Comments to Theorem 2.}

(i) We point out that the parameters of $u(t)$ depend only on $h, k_{0}$, and the chosen constants $c, \varepsilon, m$ and do not depend on the function $\tau_{0}(t)$ from (4); i.e., our feedback $-K(t) \cdot \operatorname{sign} x(t-\tau(t))$ is robust with respect to an uncertain variable delay $\tau(t)$, as well as a deviation of $F(x, t)$ in the framework of restrictions (2), (7), (5), (25), (21).

(ii) If $m \rightarrow \infty$ and $\varepsilon \rightarrow 0$ in Theorem 2 , then

$$
q^{m} \rightarrow \delta \frac{\alpha \lambda_{1}\left(1-e^{-\theta \beta / \alpha}\right)+\beta}{\left(2 e^{-\lambda_{1} h}-1\right) \beta-\alpha \lambda_{1}\left(1-e^{-\theta \beta / \alpha}\right)},
$$

and hence, the right-hand side of (36) tends to

$$
K_{0} \delta \triangleq \frac{2\left(\alpha \lambda_{1}\left(1-e^{-\theta \beta / \alpha}\right)+\beta\left(1-e^{-\lambda_{1} h}\right)\right)}{k_{0}\left(\left(2 e^{-\lambda_{1} h}-1\right) \beta-\alpha \lambda_{1}\left(1-e^{-\theta \beta / \alpha}\right)\right)} \delta .
$$

(iii) The hypotheses (14), (25), and (30) in Theorem 2 contain restrictions to the parameters of system (1), (3) in an implicit form. However, one can in principle extract some explicit conditions from them. First of all, given $\alpha$ and $\beta$, the parameter $k_{0}$ must satisfy (25) for $\delta=0$. Suppose now that such $\alpha, \beta$, and $k_{0}$ are fixed, and describe $\delta$ which meet the hypotheses (14), (25), and (30).

Condition (14) is an explicit upper bound to $\delta$.

In turn, $k_{\min }=k_{\min }(\delta)$ in (25) is a strictly decreasing function of $\delta$ (indeed, when $\delta$ grows, the allowed range for $k_{0}$ must shrink), and this function is given by (21) and (24). That is, (25) also can be written as an upper bound $\delta<\left(k_{\min }\right)^{-1}\left(k_{0}\right)$. 
At last, condition (30) after removing $\varepsilon$ (which can be arbitrarily small positive) reduces to inequality (23), where $\theta$ comes from (21). It is not difficult to show that the latter equation defines $\theta$ as a strictly decreasing function of $\delta$. Inequality (23) holds for $\delta=0$, since this is equivalent to the above assumption $k_{0}<\left.k_{\min }\right|_{\delta=0}$. Thus, the left-hand side of (23) is a positive function of $\delta$, whereas the right-hand side drops from positive to negative as $\delta$ goes from zero to $\infty$. Equating both sides of (23), we then obtain the minimal positive root $\delta_{\min }$ and finally reduce condition (30) to an upper bound $\delta<\delta_{\min }$.

4. Control algorithm. We shortly describe how to apply Theorems 1 and 2 . One begins with a few common initial steps:

1. Given system (1), (3), obeying (2), (4), and (7) with known $h>0$ and $\delta \geq 0$, we start by solving simultaneously the equations

$$
\begin{aligned}
\frac{\alpha(1+\delta)}{\beta^{2}}\left(1-e^{-\theta_{m} \beta / \alpha}\right) & =\frac{1+\delta}{\beta} \theta_{m}-\frac{1-\delta}{k_{m}}, \\
\frac{1+\delta}{\beta}\left(1-e^{-\theta_{m} \beta / \alpha}\right) & =\frac{2 e^{-\lambda_{1}\left(k_{m}\right) h}-1-\delta}{\alpha \lambda_{1}\left(k_{m}\right)}
\end{aligned}
$$

with respect to positive unknowns $k_{m}$ and $\theta_{m}$.

2. Take the solution $\left(k_{m}, \theta_{m}\right)$, and verify that the given function $F(x, t)$ satisfies (5) with certain positive $k_{0}<k_{m}$; then find the positive root $\theta$ of (21).

3. Compute the roots $\lambda_{1}>0>\lambda_{2}$ of the characteristic equation, and check the validity of (14).

4.1. Perturbations that vanish at the origin. Perform steps $1-3$ as described above and then do the following.

4. Pick a constant $c$, satisfying (28), and compute the values of $\xi\left(\sigma_{\max }\right), t_{c}$, $t_{\xi\left(\sigma_{\max }\right)}$, and $\rho$ using the formulas of Theorem 1. Verify that the initial function $\varphi$ belongs to $\Phi_{c}$ as described in Definition 3.1.

Remark 3. It is possible to set a limit $n^{*}$ to the maximum number of allowed switches of the controller or to the time interval $t \leq t^{*}$, when switches are allowed. Pick $n \leq n^{*}$ or $n \leq\left(t^{*}-t_{c}\right) / t_{\xi\left(\sigma_{\max }\right)}$, respectively. The solution becomes bounded by $|x(t)| \leq \rho^{n} / k_{0}$ after $t \geq t^{*}$.

4.2. Perturbations that do not vanish at the origin. Again perform steps $1-3$ as above, and then proceed in the following way.

4. Pick a positive $\varepsilon$ satisfying (30), and compute $T(\varepsilon)$ by (33).

5. For the last step there are three possibilities:

(a) Choose an upper bound $m^{*}$ to the number of allowed switches of the controller, and pick $m \leq m^{*}$.

(b) Set the size $t^{*}$ of the time interval when switches are allowed, and pick $m \leq t^{*} / T(\varepsilon)$.

In both cases solve (31) with respect to $q$. The solution will be bounded according to (36).

(c) In this case we bring the solution to the $\delta\left(K_{0}+\kappa\right)$-neighborhood of zero, where $K_{0}$ is taken from (37), and $\kappa$ is a (relatively) small prescribed positive parameter. Using (31) we compute

$$
q=\frac{B_{1}+B_{2}}{C+B_{2}-(1-\varepsilon) /\left(k_{0}\left(K_{0}+\kappa\right)+1\right)},
$$

Copyright $@$ by SIAM. Unauthorized reproduction of this article is prohibited. 
where

$$
B_{1}=\frac{1-e^{-\theta \beta / \alpha}}{\beta}, \quad B_{2}=\frac{\left(e^{\lambda_{1} h}-e^{\lambda_{2} h}\right)}{\alpha\left(\lambda_{1} e^{\lambda_{1} h}-\lambda_{2} e^{\lambda_{2} h}\right)}, \quad C=\frac{2 e^{-\lambda_{1} h}-1-\varepsilon}{\alpha \lambda_{1}},
$$

and, finally, put

$$
m=\left[\frac{\log \left(\delta\left(k_{0}\left(K_{0}+\kappa\right)+1\right) /(1-\varepsilon)\right)}{\log q}\right]+1 .
$$

5. Numerical example: Stabilization of an inverted pendulum. Consider the stabilization problem of an inverted pendulum via a controller with uncertain delay. The oscillations of an inverted pendulum with unit mass with such a controller are described by

$$
\ddot{x}+k \dot{x}-p \sin x+\delta=u(t-\tau(t)),
$$

where $k>0$ is a friction coefficient, $p=g / l>0, \delta$ is uncertainty, and $\tau$ is an uncertain time delay $0<\tau_{0}(t) \leq \tau(t) \leq h$. Consider the case when $k=1$ and $p=g / l=1$.4. In this case (39) takes the form

$$
\ddot{x}(t)=-\dot{x}(t)+1.4 \sin (x)+\delta-K(t) \operatorname{sign}(x(t-\tau(t))),
$$

with $\tau=0.05+0.04 \sin (t)$. It is clear that

$$
\alpha=\beta=1 \quad \text { and } \quad F(x, t)=1.4 \sin (x)+\delta
$$

and that the bound

$$
0<\tau_{0}(t) \leq \tau(t) \leq h=0.1
$$

holds.

5.1. Application of Theorem $1(\delta=0)$. The solution to $(38)$ is $k_{m}=$ 2.69518, $\theta_{m}=1.00498$. A possible $k_{0}<k_{m}$ that satisfies (5) is $k_{0}=1.5$. For that $k_{0}$ we use (21) to obtain $\theta=1.42652$. The roots of the characteristic equation are

$$
\lambda_{1}=0.82288 \text { and } \quad \lambda_{2}=-1.82288
$$

The validity of (14) is easily verified: $\delta=0<2 e^{-\lambda_{1} h}-1=0.84301$.

Now we pick a constant $c$ satisfying (28)

$$
c=1<\frac{2 e^{-\lambda_{1} h}-1}{\alpha \lambda_{1}}=1.0233
$$

Next, we have

$$
\xi\left(\sigma_{\max }\right)=\frac{1-e^{-\theta \beta / \alpha}}{\beta}=0.7599, \quad t_{c}=5.26, \quad t_{\xi\left(\sigma_{\max }\right)}=2.294, \quad \rho=0.837 .
$$

Figure 3 shows the response of the system when the initial conditions are set to $\dot{x}(-h)=1$ and $x(-h)=0.05$.

Copyright (c) by SIAM. Unauthorized reproduction of this article is prohibited. 

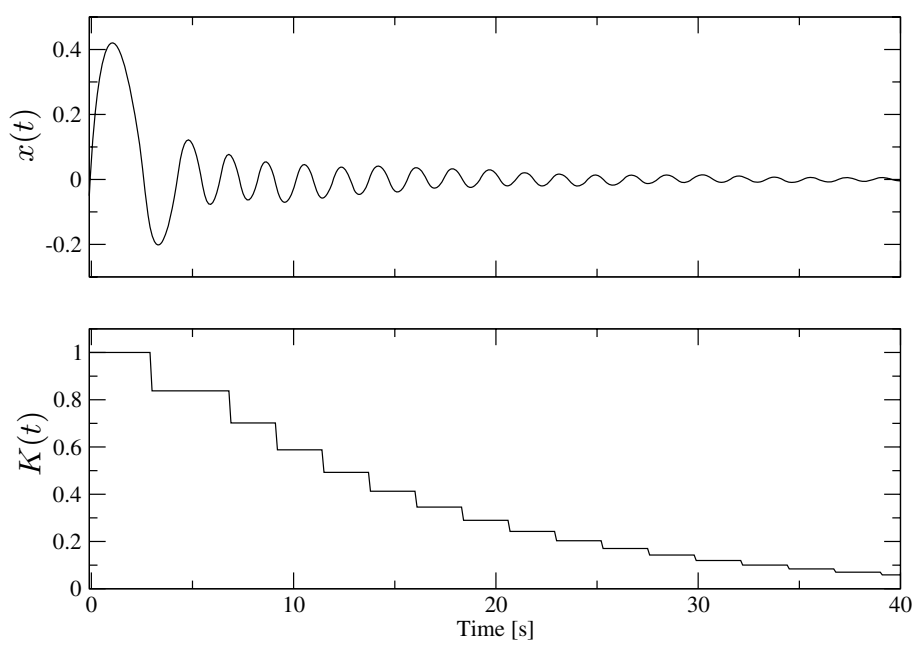

FIG. 3. System's response, $\delta=0$.
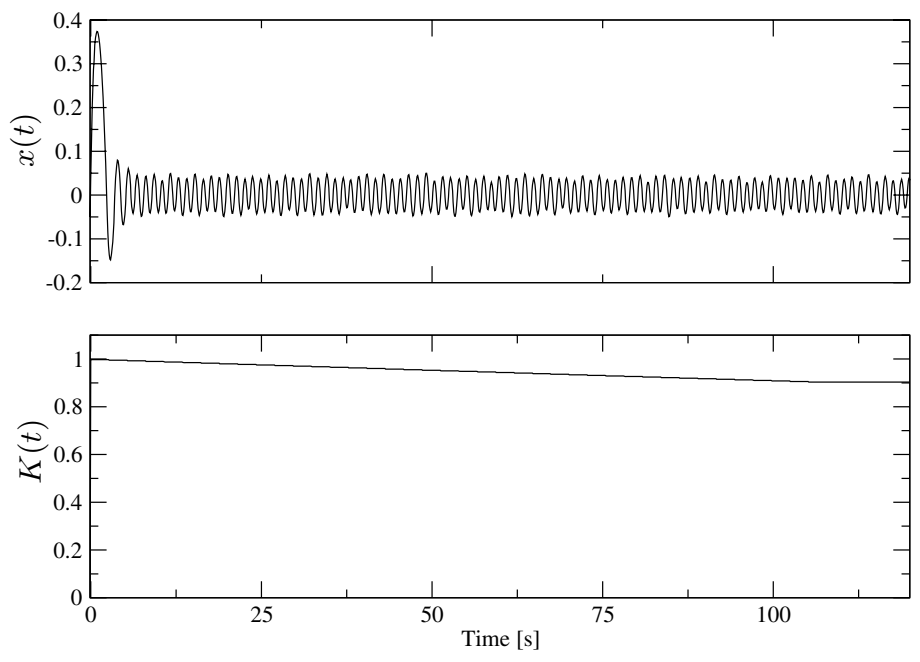

FIG. 4. System's response, $\delta=0.05$.

5.2. Application of Theorem $2(\delta=0.05)$. The following parameters were obtained as in the previous section:

$$
\begin{gathered}
k_{m}=2.2854, \quad k_{0}=1.5, \quad \lambda_{1}=0.8229 \\
\theta_{m}=1.0438, \quad \theta=1.3418, \quad \lambda_{2}=-1.8229 \\
\delta<2 e^{-\lambda_{1} h}-1=0.7930
\end{gathered}
$$

Now we pick an $\varepsilon=0.15$ satisfying (30) and evaluate $T(\varepsilon)=2.696$. For the last step we choose $m=m^{*}=40$ and obtain $q=0.9975$.

The results are shown in Figure 4. The initial data were $\dot{x}(-h)=1$ and $x(-h)=$ -0.05 .

Copyright (c) by SIAM. Unauthorized reproduction of this article is prohibited. 
6. Conclusions. The dynamics of the second order systems with a delayed relay control is analyzed. Sufficient conditions for robust delayed relay semiglobal stabilization of second order systems are found. Such conditions relate to the upper bound of an uncertainty in time delay and the parameters of the plant. An algorithm for a delayed relay control with gain adaptation is suggested. The algorithm is based on delayed information about the sign of the controlled variable only. The proposed algorithm suppresses bounded uncertainties in the time delay: Once being designed for the upper bound of time delay in the given system, this control law ensures semiglobal stabilization for any constant or variable time delay within the given constraint.

\section{Appendix A. Proofs.}

A.1. Preliminary estimates. In what follows we always suppose that $\tau(t)$ and $F(x, t)$ satisfy restrictions (4) and (7), respectively.

Lemma A.1. (i) Let

$$
0<a<b<\frac{2 e^{-\lambda_{1} h}-1}{\alpha \lambda_{1}}
$$

Then

$$
\begin{aligned}
& y_{a}(t) \leq \rho(a, b) y_{b}(t), \quad \dot{y}_{0, a}(t) \leq \rho(a, b) \dot{y}_{0, b}(t), \quad 0 \leq t \leq h, \\
& z_{a}(t) \leq \rho(a, b) z_{b}(t), \quad \dot{z}_{0, a}(t) \leq \rho(a, b) \dot{z}_{0, b}(t), \quad h \leq t \leq t_{a},
\end{aligned}
$$

with $\rho(a, b)$ defined by $(26)$.

(ii) Let $0<q<1$ and $a \geq 0$. Put

$$
\delta_{1}=\frac{\delta}{q}, \quad a_{1}=\frac{a}{q}+\frac{(1-q)\left(e^{\lambda_{1} h}-e^{\lambda_{2} h}\right)}{q \alpha\left(\lambda_{1} e^{\lambda_{1} h}-\lambda_{2} e^{\lambda_{2} h}\right)} .
$$

Then

$$
\begin{gathered}
q^{-1} y_{\delta, a}(t) \leq y_{\delta_{1}, a_{1}}(t), \quad q^{-1} \dot{y}_{\delta, a}(t) \leq \dot{y}_{\delta_{1}, a_{1}}(t), \quad 0 \leq t \leq h, \\
q^{-1} z_{\delta, a}(t) \leq z_{\delta_{1}, a_{1}}(t), \quad q^{-1} \dot{z}_{\delta, a}(t) \leq \dot{z}_{\delta_{1}, a_{1}}(t), \quad h \leq t \leq t_{\delta, a} .
\end{gathered}
$$

Proof. (i) The first inequality in (A.1) follows from the second one, and, respectively, the first inequality in (A.2) follows from the second inequality and from (A.1).

The second inequality in (A.1) can be rewritten as

$$
\rho(a, b) \geq \max _{[0, h]} \frac{\dot{y}_{a}(t)}{\dot{y}_{b}(t)} .
$$

We have

$$
\frac{\dot{y}_{a}(t)}{\dot{y}_{b}(t)}=\frac{a \alpha \Sigma(t)+1}{b \alpha \Sigma(t)+1}, \quad \Sigma(t)=\frac{\lambda_{1} e^{\lambda_{1} t}-\lambda_{2} e^{\lambda_{2} t}}{e^{\lambda_{1} t}-e^{\lambda_{2} t}}
$$

Since

$$
\frac{d}{d s}\left(\frac{a \alpha s+1}{b \alpha s+1}\right)=\frac{\alpha(a-b)}{(b \alpha s+1)^{2}}<0, \quad \dot{\Sigma}(t)=-\frac{\left(\lambda_{1}-\lambda_{2}\right)^{2} e^{-t / \alpha}}{\left(e^{\lambda_{1} t}-e^{\lambda_{2} t}\right)^{2}}<0,
$$

Copyright (c) by SIAM. Unauthorized reproduction of this article is prohibited. 
we obtain in view of $(26)$

$$
\max _{[0, h]} \frac{\dot{y}_{a}(t)}{\dot{y}_{b}(t)}=\frac{\dot{y}_{b}(h)}{\dot{y}_{b}(h)}=\rho(a, b) .
$$

We start proving the second inequality in (A.2) with the observation that $t_{a}<t_{b}$. Then, in particular, $\dot{z}_{0, b}(t)>0$ as $h \leq t \leq t_{a}$. Again we have to show that

$$
\rho(a, b) \geq \max _{\left.h, t_{a}\right]} \frac{\dot{z}_{a}(t)}{\dot{z}_{b}(t)}=\max _{\left[h, t_{a}\right]} \frac{A_{1}(a) e^{\lambda_{1} t}+A_{2}(a) e^{\lambda_{2} t}}{A_{1}(b) e^{\lambda_{1} t}+A_{2}(b) e^{\lambda_{2} t}},
$$

where

$$
A_{1}(\sigma)=-\left(2 e^{-\lambda_{1} h}-1-\alpha \sigma \lambda_{1}\right), \quad A_{2}(\sigma)=2 e^{-\lambda_{2} h}-1-\alpha \sigma \lambda_{2} .
$$

Since

$$
\frac{d}{d t}\left(\frac{A_{1}(a) e^{\lambda_{1} t}+A_{2}(a) e^{\lambda_{2} t}}{A_{1}(b) e^{\lambda_{1} t}+A_{2}(b) e^{\lambda_{2} t}}\right)=\frac{\left(A_{1}(a) A_{2}(b)-A_{2}(a) A_{1}(b)\right)\left(\lambda_{1}-\lambda_{2}\right) e^{-t / \alpha}}{\left(A_{1}(b) e^{\lambda_{1} t}+A_{2}(b) e^{\lambda_{2} t}\right)^{2}}
$$

and

$$
A_{1}(a) A_{2}(b)-A_{2}(a) A_{1}(b)=\alpha(b-a)\left(\lambda_{2}\left(2 e^{-\lambda_{1} h}-1\right)-\lambda_{1}\left(2 e^{-\lambda_{2} h}-1\right)\right)<0,
$$

we derive in view of (26) that

$$
\max _{\left.h, t_{a}\right]} \frac{\dot{z}_{a}(t)}{\dot{z}_{b}(t)}=\frac{\dot{z}_{a}(h)}{\dot{z}_{b}(h)}=\rho(a, b) .
$$

(ii) It is enough to establish the second inequality both in (A.4) and in (A.5). The second inequality in (A.4) can be rewritten as

$$
q \geq \max _{[0, h]} \frac{\dot{y}_{\delta, a}(t)}{\dot{y}_{\delta_{1}, a_{1}}(t)}=\max _{[0, h]} \frac{a \alpha \Sigma(t)+1+\delta}{a_{1} \alpha \Sigma(t)+1+\delta_{1}} .
$$

Since

$$
\frac{d}{d s}\left(\frac{a \alpha s+1+\delta}{a_{1} \alpha s+1+\delta_{1}}\right)=-\frac{1-q}{q} \cdot \frac{\left.a+(1+\delta)(\alpha \Sigma(h))^{-1}\right)}{\left(a_{1} \alpha s+1+\delta_{1}\right)^{2}}<0
$$

and $\dot{\Sigma}(t)<0$, we obtain

$$
\max _{[0, h]} \frac{\dot{y}_{\delta, a}(t)}{\dot{y}_{\delta_{1}, a_{1}}(t)}=\frac{\dot{y}_{\delta, a}(h)}{\dot{y}_{\delta_{1}, a_{1}}(h)}=q
$$

The second inequality in (A.5) follows, first, from the fact that the function $q^{-1} z_{\delta, a}(t)$ solves the problem

$$
\alpha \ddot{z}=-\beta \dot{z}+k_{0} z-q^{-1}+\delta_{1}, \quad z(h)=q^{-1} y_{\delta, a}(h), \dot{z}(h)=q^{-1} \dot{y}_{\delta, a}(h),
$$

whereas the function $z_{\delta_{1}, a_{1}}(t)$ solves the problem

$$
\alpha \ddot{z}=-\beta \dot{z}+k_{0} z-1+\delta_{1}, \quad z(h)=y_{\delta_{1}, a_{1}}(h), \dot{z}(h)=\dot{y}_{\delta_{1}, a_{1}}(h),
$$

Copyright $@$ by SIAM. Unauthorized reproduction of this article is prohibited. 
where $y_{\delta_{1}, a_{1}}(h) \geq q^{-1} y_{\delta, a}(h), \dot{y}_{\delta_{1}, a_{1}}(h)=q^{-1} y_{\delta, a}(h)$, and, second, from the inequality $t_{\delta, a} \leq t_{\delta_{1}, a_{1}}$. In turn, the latter relation is an immediate consequence of (16).

Lemma A.2. (i) Assume that $t^{*}>0, t_{0} \in\left[t^{*}-h, t^{*}\right]$, and that $a \geq 0$ satisfies (15). Let a solution $x_{\varphi}(t)$ to the equation

$$
\alpha \ddot{x}(t)=-\beta \dot{x}(t)+F(x(t), t)-\operatorname{sign} x(t-\tau(t))
$$

be such that

$$
0 \leq x_{\varphi}\left(t^{*}\right) \leq y_{\delta, a}\left(t^{*}-t_{0}\right), \quad \dot{x}_{\varphi}\left(t^{*}\right) \leq \dot{y}_{\delta, a}\left(t^{*}-t_{0}\right) .
$$

Then

$$
x_{\varphi}(t) \leq y_{\delta, a}\left(t-t_{0}\right), \quad \dot{x}_{\varphi}(t) \leq \dot{y}_{\delta, a}\left(t-t_{0}\right), \quad t \in\left[t^{*}, t_{0}+h\right] .
$$

(ii) Assume that $t_{0} \geq 0$ and that $a \geq 0$ satisfies (15). Let a solution $x_{\varphi}(t)$ obey the conditions

$$
x_{\varphi}(t)>0, t \in\left(t_{0}, t_{0}+h\right], \quad x_{\varphi}\left(t_{0}+h\right) \leq z_{\delta, a}(\sigma), \quad \dot{x}_{\varphi}\left(t_{0}+h\right) \leq \dot{z}_{\delta, a}(\sigma)
$$

for some $\sigma \in\left[h, t_{\delta, a}\right]$. Then

$$
x_{\varphi}(t) \leq z_{\delta, a}\left(t-\sigma+t_{0}+h\right), \quad \dot{x}_{\varphi}(t) \leq \dot{z}_{\delta, a}\left(t-\sigma+t_{0}+h\right)
$$

for all $t \geq t_{0}+h$ such that $x_{\varphi}(t) \geq 0$.

Proof. (i) Since $y_{\delta, a}(t)$ is a strictly increasing function, it is sufficient to consider the case when $\dot{x}_{\varphi}(t) \geq 0, t \in\left[t^{*}, t_{0}+h\right]$. Then, in the interval $\left[t^{*}, t_{0}+h\right)$, we have

$$
\alpha \ddot{x}_{\varphi}(t)=-\beta \dot{x}_{\varphi}(t)+F\left(x_{\varphi}(t), t\right) \pm 1 \leq-\beta \dot{x}_{\varphi}(t)+k_{0} x_{\varphi}(t)+1+\delta,
$$

which after a double integration turns into

$$
\begin{aligned}
\dot{x}_{\varphi}(t) \leq & \dot{x}_{\varphi}\left(t^{*}\right) e^{-\beta\left(t-t^{*}\right) / \alpha}+\frac{1}{\alpha} \int_{t^{*}}^{t}\left(k_{0} x_{\varphi}(\xi)+1+\delta\right) e^{\beta(\xi-t) / \alpha} d \xi \\
x_{\varphi}(t)-x_{\varphi}\left(t^{*}\right) \leq & \frac{1+\delta}{\beta}\left(t-t^{*}\right)+\frac{\alpha}{\beta}\left(\dot{x}_{\varphi}\left(t^{*}\right)-\frac{1+\delta}{\beta}\right)\left(1-e^{-\left(t-t^{*}\right) / \alpha}\right) \\
& +\frac{k_{0}}{\beta} \int_{t^{*}}^{t} x_{\varphi}(\xi)\left(1-e^{\beta(\xi-t) / \alpha}\right) d \xi
\end{aligned}
$$

Due to the monotonicity of the right-hand sides with respect to $x_{\varphi}$ and $\dot{x}_{\varphi}$, inequality (A.7), and the fact that the substitution of $y_{\delta, a}(t)$ for $x_{\varphi}(t)$ turns (A.8) and (A.9) into equalities, we obtain subsequently that $x_{\varphi}(t) \leq y_{\delta, a}\left(t-t_{0}\right)$ and $\dot{x}_{1, \varphi}(t) \leq \dot{y}_{\delta, a} a\left(t-t_{0}\right)$, $t \in\left[t^{*}, t_{0}+h\right]$.

(ii) Let $x_{\varphi}(t)>0$ in an interval $\left[t_{0}+h, t_{1}\right)$ for some $t_{1}>t_{0}+h$. Since $x_{\varphi}(t)$ is positive in $\left(t_{0}, t_{0}+h\right]$, and $\tau(t) \leq h$, we obtain

$$
\alpha \ddot{x}_{\varphi}(t) \leq-\beta \dot{x}_{\varphi}(t)+k_{0} x_{\varphi}(t)-1+\delta, \quad t \in\left[t_{0}+h, t_{1}\right],
$$

Copyright $@$ by SIAM. Unauthorized reproduction of this article is prohibited. 
and hence

$$
\begin{aligned}
\dot{x}_{\varphi}(t) \leq & \dot{x}_{\varphi}\left(t_{0}+h\right) e^{-\beta\left(t-t_{0}-h\right) / \alpha} \\
& +\frac{1}{\alpha} \int_{t_{0}+h}^{t}\left(k_{0} x_{\varphi}(\xi)-1+\delta\right) e^{\beta(\xi-t) / \alpha} d \xi \\
x_{\varphi}(t)-x_{\varphi}\left(t_{0}+h\right) \leq & -\frac{1-\delta}{\beta}\left(t-t_{0}-h\right) \\
& +\frac{\alpha}{\beta}\left(\dot{x}_{\varphi}\left(t_{0}+h\right)+\frac{1-\delta}{\beta}\right)\left(1-e^{-\beta\left(t-t_{0}-h\right) / \alpha}\right) \\
& +\frac{k_{0}}{\beta} \int_{t_{0}+h}^{t} x_{\varphi}(\sigma)\left(1-e^{\beta(\sigma-t) / \alpha}\right) d \sigma .
\end{aligned}
$$

These relations are monotone with respect to $x_{\varphi}$ and $\dot{x}_{\varphi}$ and thereby imply $x_{\varphi}(t) \leq$ $z_{a}\left(t-\sigma+t_{0}+h\right), \dot{x}_{\varphi}(t) \leq \dot{z}_{a}\left(t-\sigma+t_{0}+h\right), t \in\left[t_{0}+h, t_{1}\right]$, since the replacement of $x_{\varphi}(t)$ by $z_{a}\left(t-\sigma+t_{0}+h\right)$ turns (A.10) and (A.11) into equalities.

Lemma A.3. For any nonnegative a satisfying (15), and any $\varphi \in C_{0}[-h, 0]$ such that

$$
\varphi(0)=0, \quad \dot{\varphi}(0)=a,
$$

the solution $x_{\varphi}(t)$ to (A.6) satisfies the following conditions:

- $x_{\varphi}(t)$ has an unbounded zero locus;

- the first positive zero of $x_{\varphi}(t)$ does not exceed $t_{\delta, a}$;

- if $x_{\varphi}\left(t^{\prime}\right)=0$ at $t^{\prime}>0$, then $x_{\varphi}$ vanishes at some $t^{\prime \prime} \in\left(t^{\prime}, t^{\prime}+t_{\delta, \xi_{\delta}\left((1-\delta) / k_{0}\right)}\right)$;

- $x_{\varphi}(t)$ obeys the condition

$$
\begin{gathered}
\left|x_{\varphi}(t)\right| \leq z_{\delta, \xi_{\delta}\left((1-\delta) / k_{0}\right)}\left(t_{\delta, \xi_{\delta}\left((1-\delta) / k_{0}\right)}\right) \quad \text { if } \quad t \geq t_{\delta, a} \\
\left|\dot{x}_{\varphi}(t)\right| \leq \xi_{\delta}\left((1-\delta) / k_{0}\right) \quad \text { for all } \quad t \in x_{\varphi}^{-1}(0) \cap(0, \infty) .
\end{gathered}
$$

Proof. Step 1. We first show that $x_{\varphi}(t)$ has a positive root. Assume on the contrary that $x_{\varphi}(t)>0$ for all $t>0$. By Lemma A.2(i), $x_{\varphi}(t) \leq y_{a}(t), \dot{x}_{\varphi}(t) \leq \dot{y}_{a}(t)$ as $t \in[0, h]$, and hence by Lemma A.2(i), $x_{\varphi}(t) \leq z_{a}(t)$ as $t \geq h$. However, $z_{a}(t)$ becomes negative for large $t$, and so does $x_{\varphi}$. Furthermore, we obtain that $x(t)$ vanishes at some point $t_{1} \leq t_{a}$, where $t_{a}$ denotes the positive zero of $z_{a}(t)$. The same argument provides the upper bound $\left|x_{\varphi}(t)\right| \leq z_{a}\left(t_{a}\right)$ in the interval $\left(0, t_{1}\right)$.

Step 2. We intend now to estimate $\left|\dot{x}_{\varphi}\left(t_{1}\right)\right|$. Change sign of $x_{\varphi}$ so that $x_{\varphi}(t)<0$ as $t \in\left(0, t_{1}\right)$. Let $t^{\prime}=\max \left\{t \in\left(0, t_{1}\right) \mid \dot{x}_{\varphi}(t)=0\right\}$. Since $\left|x_{\varphi}\left(t^{\prime}\right)\right| \leq z_{a}\left(t_{a}\right)<\frac{1}{k_{0}}$, in the interval $\left[t^{\prime}, t_{1}\right]$, we have

$$
\alpha \ddot{x}_{\varphi}(t)=-\beta \dot{x}_{\varphi}(t)+F\left(x_{\varphi}(t), t\right) \pm 1<-\dot{x}_{\varphi}(t)+1,
$$

which yields

$$
\dot{x}_{\varphi}(t)<1-e^{\left(t^{\prime}-t\right) / \alpha}<1, \quad t \in\left[t^{\prime}, t_{1}\right] .
$$

Furthermore, $x_{\varphi}(t)$ is strongly increasing in $\left[t^{\prime}, t_{1}\right]$, and then we can choose it as a variable and rewrite (A.15) in the form

$$
\alpha \frac{d \dot{x}_{\varphi}}{d x_{\varphi}} \dot{x}_{1, \varphi}+\dot{x}_{\varphi}<1 \quad \stackrel{0 \leq \dot{x}_{\varphi}<1}{=} \quad \int_{0}^{\dot{x}_{\varphi}\left(t_{1}\right)} \frac{\alpha \dot{x}_{\varphi}}{1-\dot{x}_{\varphi}} d \dot{x}_{1, \varphi}<-x_{\varphi}\left(t^{\prime}\right) .
$$

Copyright (c) by SIAM. Unauthorized reproduction of this article is prohibited. 
The latter formula turns into the relation for $\xi\left(1 / k_{0}\right)$, when replacing " $<$ " by "=" and $-x_{\varphi}\left(t^{\prime}\right)$ by $\frac{1}{k_{0}}$. Hence $\dot{x}_{\varphi}\left(t_{1}\right)<\xi\left(1 / k_{0}\right)$.

Step 3. Observe now that (23) is equivalent to

$$
\xi\left(\frac{1}{k_{0}}\right)<\frac{2 e^{-\lambda_{1} h}-1}{\alpha \lambda_{1}} .
$$

That is, $x_{\varphi}\left(t-t_{1}\right)$ satisfies the hypotheses of Lemma A.3, and one can proceed inductively, proving the statements of the lemma for the whole interval $[0, \infty)$.

A.2. Proof of Theorem 1. In the interval $\left[0, t_{c}+t_{\xi\left(1 / k_{0}\right)}\right]$, we have $x_{u, \varphi}(t)=$ $x_{\varphi}(t)$. Hence the conditions imposed on the set $\Phi_{c} \subset C_{0}[-h, 0]$ and Lemmas A.2 and A.3 yield that $\left|x_{u, \varphi}(t)\right| \leq \frac{1}{k_{0}}$ as $t \in\left[0, t_{c}\right]$ and $\left(x_{u, \varphi}\right)^{-1}(0) \cap\left(0, t_{c}\right] \neq \emptyset$. Furthermore, for any $t^{*} \in\left(x_{u, \varphi}\right)^{-1}(0) \cap\left(0, t_{c}\right]$, we have $\left|\dot{x}_{u, \varphi}\left(t^{*}\right)\right| \leq \xi\left(1 / k_{0}\right)$. Next we apply Lemmas A.2 and A.3 to $x_{u, \varphi}$ restricted to the interval $\left[t_{c}, t_{c}+t_{\xi\left(1 / k_{0}\right)}\right]$ and obtain that $\left|x_{u, \varphi}(t)\right| \leq z_{\xi\left(1 / k_{0}\right)}\left(t_{\xi\left(1 / k_{0}\right)}\right)$ if $t \in\left[t_{c}, t_{c}+t_{\xi\left(1 / k_{0}\right)}\right]$, the set $\left(x_{u, \varphi}\right)^{-1}(0) \cap\left(t_{c}, t_{c}+t_{\xi\left(1 / k_{0}\right)}\right]$ is nonempty, and, for any $t^{*} \in\left(x_{u, \varphi}\right)^{-1}(0) \cap\left(t_{c}, t_{c}+t_{\xi\left(1 / k_{0}\right)}\right]$, it holds that $\left|\dot{x}_{u, \varphi}\left(t^{*}\right)\right| \leq$ $\xi\left(z_{\xi\left(1 / k_{0}\right)}\left(t_{\xi\left(1 / k_{0}\right)}\right)\right)$.

In the interval $\left[t_{c}+t_{\xi\left(1 / k_{0}\right)}, t_{c}+2 t_{\xi\left(1 / k_{0}\right)}\right]$, we have

$$
\alpha \ddot{x}_{u, \varphi}(t)=-\beta \dot{x}_{u, \varphi}+F\left(x_{u, \varphi}(t), t\right)-\rho \cdot \operatorname{sign} x_{u, \varphi}(t-\tau(t)) .
$$

The variable change $x_{u, \varphi}(t)=\rho \cdot x_{u, \varphi}^{(1)}(t), t \in\left[t_{c}+t_{\xi\left(1 / k_{0}\right)}, t_{c}+2 t_{\xi\left(1 / k_{0}\right)}\right]$, leads to the equation

$$
\alpha \frac{d^{2}}{d t^{2}} x_{u, \varphi}^{(1)}(t)=-\beta \frac{d}{d t} x_{u, \varphi}^{(1)}(t)+\frac{1}{\rho} F\left(\rho x_{u, \varphi}^{(1)}(t), t\right)-\operatorname{sign} x_{u, \varphi}^{(1)}(t-\tau(t)) .
$$

Observe that the function $F^{(1)}(x, t):=\frac{1}{\rho} F(\rho x, t)$ satisfies restrictions (2) and (5).

Put

$$
s=\max \left(\left(x_{u, \varphi}\right)^{-1}(0) \cap\left[0, t_{c}+t_{\xi\left(1 / k_{0}\right)}\right]\right) .
$$

Assume, first, that $s>t_{c}+t_{\xi\left(1 / k_{0}\right)}-h$. Then by Lemma A.2(i)

$$
\begin{aligned}
& \left|x_{u, \varphi}\left(t_{c}+t_{\xi\left(1 / k_{0}\right)}\right)\right| \leq y_{\xi\left(z_{\xi\left(1 / k_{0}\right)}\left(t_{\xi\left(1 / k_{0}\right)}\right)\right)}\left(t_{c}+t_{\xi\left(1 / k_{0}\right)}-s\right), \\
& \left|\dot{x}_{u, \varphi}\left(t_{c}+t_{\xi\left(1 / k_{0}\right)}\right)\right| \leq \dot{y}_{\xi\left(z_{\xi\left(1 / k_{0}\right)}\left(t_{\xi\left(1 / k_{0}\right)}\right)\right)}\left(t_{c}+t_{\xi\left(1 / k_{0}\right)}-s\right) .
\end{aligned}
$$

Consequently, by Lemma A.1(i) and by the definition of $\rho$,

$$
\begin{aligned}
\left|x_{u, \varphi}^{(1)}\left(t_{c}+t_{\xi\left(1 / k_{0}\right)}\right)\right| & \leq y_{\xi\left(1 / k_{0}\right)}\left(t_{c}+t_{\xi\left(1 / k_{0}\right)}-s\right), \\
\left|\frac{d}{d t} x_{u, \varphi}^{(1)}\left(t_{c}+t_{\xi\left(1 / k_{0}\right)}\right)\right| & \leq \dot{y}_{\xi\left(1 / k_{0}\right)}\left(t_{c}+t_{\xi\left(1 / k_{0}\right)}-s\right) ;
\end{aligned}
$$

that is, $x_{u, \varphi}^{(1)}\left(t-t_{c}-t_{\xi\left(1 / k_{0}\right)}\right)$ satisfies the conditions of Lemmas A.2(i) and A.3 with $a=\xi\left(1 / k_{0}\right)$. A.2(ii)

Now assume that $s$ from (A.16) satisfies $s \leq t_{c}+t_{\xi\left(1 / k_{0}\right)}-h$. Then by Lemma

$$
\begin{aligned}
& \left|x_{u, \varphi}\left(t_{c}+t_{\xi\left(1 / k_{0}\right)}\right)\right| \leq z_{\xi\left(z_{\xi\left(1 / k_{0}\right)}\left(t_{\xi\left(1 / k_{0}\right)}\right)\right)}(\sigma), \\
& \left|\dot{x}_{u, \varphi}\left(t_{c}+t_{\xi\left(1 / k_{0}\right)}\right)\right| \leq \dot{z}_{\xi\left(z_{\xi\left(1 / k_{0}\right)}\left(t_{\xi\left(1 / k_{0}\right)}\right)\right)}(\sigma)
\end{aligned}
$$

Copyright (c) by SIAM. Unauthorized reproduction of this article is prohibited. 


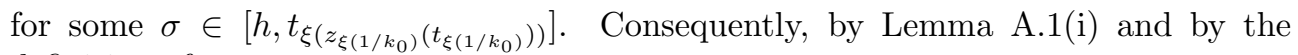
definition of $\rho$,

$$
\left|x_{u, \varphi}^{(1)}\left(t_{c}+t_{\xi\left(1 / k_{0}\right)}\right)\right| \leq z_{\xi\left(1 / k_{0}\right)}(\sigma), \quad\left|\frac{d}{d t} x_{u, \varphi}^{(1)}\left(t_{c}+t_{\xi\left(1 / k_{0}\right)}\right)\right| \leq \dot{z}_{\xi\left(1 / k_{0}\right)}(\sigma),
$$

and here $h \leq \sigma \leq t_{\xi\left(z_{\xi\left(1 / k_{0}\right)}\left(t_{\xi\left(1 / k_{0}\right)}\right)\right)}<t_{\xi\left(1 / k_{0}\right)}$. Hence $x_{u, \varphi}^{(1)}\left(t-t_{c}-t_{\xi\left(1 / k_{0}\right)}\right)$ satisfies the conditions of Lemmas A.2(ii) and A.3 with $a=\xi\left(1 / k_{0}\right)$.

Under both of the assumptions, Lemmas A.2 and A.3 yield that

$$
\left|x_{u, \varphi}^{(1)}(t)\right| \leq z_{\xi\left(1 / k_{0}\right)}\left(t_{\xi\left(1 / k_{0}\right)}\right), \quad t \in\left[t_{c}+t_{\xi\left(1 / k_{0}\right)}, t_{c}+2 t_{\xi\left(1 / k_{0}\right)}\right],
$$

the set $\left(x_{u, \varphi}^{(1)}\right)^{-1}(0) \cap\left(t_{c}+t_{\xi\left(1 / k_{0}\right)}, t_{c}+2 t_{\xi\left(1 / k_{0}\right)}\right]$ is nonempty, and

$$
\left|\frac{d}{d t} x_{u, \varphi}^{(1)}\left(t^{*}\right)\right| \leq \xi\left(z_{\xi\left(1 / k_{0}\right)}\left(t_{\xi\left(1 / k_{0}\right)}\right)\right)
$$

as $t^{*} \in\left(x_{u, \varphi}^{(1)}\right)^{-1}(0) \cap\left(t_{c}+t_{\xi\left(1 / k_{0}\right)}, t_{c}+2 t_{\xi\left(1 / k_{0}\right)}\right]$. These properties of $\left.x_{u, \varphi}^{(1)}\right|_{\left[t_{c}+t_{\xi\left(1 / k_{0}\right)}\right.}$, $\left.t_{c}+2 t_{\xi\left(1 / k_{0}\right)}\right]$ coincide with the aforementioned properties of $\left.x_{u, \varphi}\right|_{\left[t_{c}, t_{c}+t_{\xi\left(1 / k_{0}\right)}\right]}$. Thus, one can proceed inductively, defining

$$
x_{u, \varphi}(t)=\rho^{n} x_{u, \varphi}^{(n)}(t), \quad t \in\left[t_{c}+n t_{\xi\left(1 / k_{0}\right)}, t_{c}+(n+1) t_{\xi\left(1 / k_{0}\right)}\right], \quad n=2,3, \ldots,
$$

and deriving

$$
\left|x_{u, \varphi}^{(n)}(t)\right| \leq z_{\xi\left(1 / k_{0}\right)}\left(t_{\xi\left(1 / k_{0}\right)}\right), \quad t \in\left[t_{c}+n t_{\xi\left(1 / k_{0}\right)}, t_{c}+(n+1) t_{\xi\left(1 / k_{0}\right)}\right] .
$$

The upper bound (29) follows immediately.

A.3. Proof of Theorem 2. In the interval $[0, T(\varepsilon)]$, we have $x_{u, \varphi}(t)=x_{1, \varphi}(t)$. The conditions imposed on the set $\Phi_{c} \subset C_{0}[-h, 0]$ and Lemmas A.2 and A.3 yield that $\left|x_{\varphi}(t)\right| \leq \frac{1-\delta-\varepsilon}{k_{0}}$ as $t \geq 0$ and that $\left(x_{u, \varphi}\right)^{-1}(0) \cap\left(0, t_{\delta, c}\right] \neq \emptyset$.

Indeed, using (13) for the equation $z_{\delta, c}\left(t_{\delta, c}\right)=0$, we obtain

$$
\begin{gathered}
\frac{2 e^{-\lambda_{1} h}-1-\delta-\alpha c \lambda_{1}}{\alpha \lambda_{1}\left(\lambda_{1}-\lambda_{2}\right)} e^{\lambda_{1} t_{\delta, c}}<\frac{1-\delta}{k_{0}}, \\
t_{\delta, c}<\frac{1}{\lambda_{1}} \log \frac{(1-\delta) \alpha \lambda_{1}\left(\lambda_{1}-\lambda_{2}\right)}{k_{0}\left(2 e^{-\lambda_{1} h}-1-\delta-\alpha c \lambda_{1}\right)}=\frac{1}{\lambda_{1}} \log \frac{(1-\delta)\left(\lambda_{1}-\lambda_{2}\right)}{-\lambda_{2} \varepsilon}=T(\varepsilon)
\end{gathered}
$$

(cf. (33) and (35)). Furthermore, we have $\left|\dot{x}_{\varphi}\left(t^{*}\right)\right|<\xi_{\delta}\left((1-\delta) / k_{0}\right)$ for any $t^{*} \in$ $\left(x_{\varphi}\right)^{-1}(0) \cap(0, T(\varepsilon)]$. That means $x_{\varphi}(T(\varepsilon))$ and $\dot{x}_{\varphi}(T(\varepsilon))$ satisfy the hypotheses of Lemma A.2(i) or (ii) with $a=\xi_{\delta}\left(\sigma_{\delta}\right), \sigma_{\delta}$ being defined by (17). Furthermore, using (20) and (22), we obtain

$$
\begin{aligned}
a=\xi_{\delta}\left(\sigma_{\delta}\right) & =\frac{1+\delta}{\beta}\left(1-e^{-t^{\prime} \beta / \alpha}\right)=\frac{\beta}{\alpha}\left(\frac{1+\delta}{\beta} t^{\prime}-\sigma_{\delta}\right) \\
>\frac{\beta}{\alpha} & \left(\frac{1+\delta}{\beta} t^{\prime}-\frac{1-\delta}{k_{0}}\right) \quad \Longrightarrow \quad t^{\prime}<\theta \\
& \Longrightarrow a<\frac{1+\delta}{\beta}\left(1-e^{-\theta \beta / \alpha}\right) .
\end{aligned}
$$

Copyright (c) by SIAM. Unauthorized reproduction of this article is prohibited. 
In the interval $[T(\varepsilon), 2 T(\varepsilon))$, we have $K(t)=q$, and the variable change $x_{u, \varphi}(t)=$ $q x^{(1)}(t)$ leads to the equation

$$
\alpha \frac{d^{2}}{d t^{2}} x^{(1)}(t)=-\beta \frac{d}{d t} x^{(1)}(t)+F_{1}\left(x^{(1)}(t), t\right)-\operatorname{sign} x^{(1)}(t-\tau(t)), \quad t \in[T(\varepsilon), 2 T(\varepsilon)),
$$

where $F_{1}(x, t):=q^{-1} F(q x, t)$ obeys restriction (5) and restriction (7) with $\delta$ replaced by $\delta_{1}=\delta / q$. In view of (A.17) and by Lemma A.1(ii), $x^{(1)}(t)$ satisfies the conditions of Lemma A.2 with $F, \delta, a$ replaced, respectively, by $F_{1}, \delta_{1}, a_{1}$ defined in (A.3). Moreover,

$$
\begin{gathered}
a_{1}=\frac{a}{q}+\frac{(1-q)\left(e^{\lambda_{1} h}-e^{\lambda_{2} h}\right)}{q \alpha\left(\lambda_{1} e^{\lambda_{1} h}-\lambda_{2} e^{\lambda_{2} h}\right)}<\frac{1+\delta}{q \beta}\left(1-e^{-\theta \beta / \alpha}\right)+\frac{(1-q)\left(e^{\lambda_{1} h}-e^{\lambda_{2} h}\right)}{q \alpha\left(\lambda_{1} e^{\lambda_{1} h}-\lambda_{2} e^{\lambda_{2} h}\right)} \\
\quad \leq \frac{2 e^{-\lambda_{1} h}-1-\delta / q-\varepsilon}{\alpha \lambda_{1}}=\frac{2 e^{-\lambda_{1} h}-1-\delta_{1}-\varepsilon}{\alpha \lambda_{1}}
\end{gathered}
$$

the first inequality following from (A.17) and the second one from (32). We obtain also that

$$
\left|x_{u, \varphi}(t)\right|=q\left|x^{(1)}(t)\right| \leq q \frac{1-\delta_{1}}{k_{0}}=\frac{q-\delta}{k_{0}} .
$$

Relation (A.18) allows one to continue the procedure inductively by defining $K(t)$ by (34) and the functions $x^{(s)}(t), t \geq s T(\varepsilon)$, by the formula $x_{u, \varphi}(t)=q^{s} x^{(s)}(t)$ for $s=1, \ldots, m$. Inequality (36) follows immediately.

\section{REFERENCES}

[1] M. Akian M., P.-A. Bliman, And M. Sorine, Control of delay systems with relay, IMA J. Math. Control Inform., 19 (2002), pp. 133-155.

[2] G. Bartolini, G. M. Cocoli, and A. Ferrara, Vibration damping and the second-order sliding modes in the control of single finger of the AMADEUS gripper, Internat. J. Systems Sci., 29 (1998), pp. 497-512.

[3] D. Barton, B. Krauskopf, And R. E. Wilson, Explicit periodic solutions in a model of a relay controller with delay and forcing, Nonlinearity, 18 (2005), pp. 2637-2656.

[4] A. F. Filippov, Differential Equations with Discontinuous Right-Hand Side, Kluwer, Dordrecht, 1988.

[5] E. Fridman, L. Fridman, And E. Shustin, Steady modes and stability in discontinuous delay systems with periodic disturbances, ASME J. Dynam. Syst. Measurement and Control, 122 (2000), pp. 732-737.

[6] L. Fridman, E. Fridman, And E. Shustin, Steady modes and sliding modes in relay control systems with delay, in Sliding Mode Control in Engineering, J. P. Barbot and W. Perruquetti, eds., Marcel Dekker, New York, 2002, pp. 263-293.

[7] L. Fridman, V. Strygin, And A. Polyakov, Stabilization of oscillations amplitudes via relay delay control, Internat. J. Control, 76 (2003), pp. 770-780.

[8] L. Fridman, V. Strygin, and A. Polyakov, Nonlocal stabilization via delayed relay control rejecting uncertainty in a time delay, Internat. J. Robust Nonlinear Control, 14 (2004), pp. $15-37$.

[9] J. K. Hale and S. M. Verduyn Lunel, Introduction to Functional Differential Equations, Springer, New York, 1993.

[10] L. LevaGGI, Infinite dimensional systems sliding motions, Eur. J. Control, 8 (2003), pp. 508516.

[11] L. Levaggi and E. Punta, Analysis of a second-order sliding-mode algorithm in presence of input delays, IEEE Trans. Automat. Control, 51 (2006), pp. 1325-1332.

[12] A. Longtin And J. G. Milton, Modelling autonomous oscillations in the human pupil light reflex using nonlinear delay-differential equation, Bull. Math. Biol., 51 (1989), pp. 605-624.

Copyright $@$ by SIAM. Unauthorized reproduction of this article is prohibited. 
[13] A. Longtin And J. G. Milton, Insight into the transfer function, gain, and oscillation onset for the pupil light reflex using nonlinear delay-differential equations, Biol. Cybern., 61 (1989), pp. 51-58.

[14] Yu. Orlov, M. Perruquetti, and J. P. Richard, Sliding mode control synthesis of uncertain time-delay systems, Asian J. Control, 5 (2003), pp. 568-577.

[15] W. B. Ribbens, Understanding Automotive Electronics, 6th ed., Newnes, New York, 2003.

[16] Yu. Shtessel, A. Zinober, And I. Shkolnikov, Sliding mode for nonlinear systems with output delay via method of stable system center, ASME J. Dynam. Syst. Measurement and Control, 125 (2003), pp. 253-257.

[17] E. Shustin, E. Fridman, And L. Fridman, Oscillations in a second-order discontinuous system with delay, Discrete Contin. Dyn. Syst., 9 (2003), pp. 339-358.

[18] E. Shustin, Exponential decay of oscillations in a multidimensional delay differential system, in Proceedings of the 4th International Conference on Dynamical Systems and Differential Equations, Wilmington, NC, 2002, American Institute of Mathematical Sciences, Springfield, MO, 2002, pp. 809-816.

[19] V. I. UtKin, Sliding Modes in Control Optimization, Springer, Berlin, 1992.

Copyright (c) by SIAM. Unauthorized reproduction of this article is prohibited. 